\title{
28 Research Suare \\ River Branch Inspired Janus Textile with Moisture Wicking and UV Resistant Properties
}

\author{
Jinju Zhang \\ Jiangnan University \\ Juanjuan Sun \\ Jiangnan University \\ Yunjie Yin \\ Jiangnan University \\ Chaoxia Wang ( $\nabla$ wangchaoxia@sohu.com ) \\ Jiangnan University https://orcid.org/0000-0001-6322-7606
}

\section{Research Article}

Keywords: River branch, Cotton fabric, Moisture wicking, Nano-ZnO, Thermal conductivity

Posted Date: April 26th, 2021

DOI: https://doi.org/10.21203/rs.3.rs-390998/v1

License: (1) (1) This work is licensed under a Creative Commons Attribution 4.0 International License. Read Full License 


\section{Abstract}

Discontinuous hydrophobic/hydrophilic Janus fabric resembling river branches is prepared by nano-ZnO pretreatment and screen printing for directional water transport and thermal conductivity properties. A river branchlike structure is constructed dexterously, and the effect is quantitatively adjusted by accurately regulating the gap width of hexagon water-transfer-channel. Fabrics based on these rivers branch-like hexagon structure possess enhanced moisture wicking performance, a desired water transport index of $147.26 \%$, which are over seven times higher than conventional cotton fabrics. Meanwhile, nano-ZnO treatment improves the thermal conductivity of cotton fabric, which accelerates the evaporation of sweat on the surface of cotton fabric. Moreover, nano-ZnO cotton fabric shows an outstanding UV resistant property with 145 UPF value. The novel discontinuous hydrophobic/hydrophilic Janus fabric could provide a new strategy for the development of moisture management textiles.

\section{Introduction}

Fabrics with directional water transport property have attracted increasing attention due to their capability of providing a comfortable microclimate to wearers who work in extreme hot or cold environment, such as soldiers, athletes, couriers and industrial workers.(Wang et al. 2016b) Directional water transport property(Zhou et al. 2016) relies on the differential capillary effect,(Parada et al. 2017) which pulls the sweat away from human skin through tiny capillary pores by chemical forces or surface tension.(Shou and Fan 2018) Notably, the unique directional water transport property has been developed in the fields of smart textiles,(Bai et al. 2012; He et al. 2020) water harvesting,(Zhang et al. 2017; Zhu et al. 2020) oil/water separation,(Wang et al. 2016a; Li et al. 2018; Yang et al. 2019) medical and so on.(Wang et al. 2019)

Traditional hydrophilic fabric such as cotton are easily wetted by sweat, resulting in uncomfortable clamminess and colds.(Neves et al. 2017) On the contrary, hydrophobic fabric such as polyester are waterproof, (Wang et al. 2019)but it also prevents sweat from evaporating in inner side.(su et al. 2007) In recent years, there are several commercial textiles which provide different levels of directional water transport property. Specifically, the unique four-groove structure makes the Coolmax polyester fiber developed by DuPont have better capillary effect and water transport property.(Kim 2020) In addition, Aerocool moisture wicking fiber developed by Korea Hyosung Corporation and the Wellkey polyester hollow fiber developed by Teijin also have certain water transport property. However, the water transport in such textiles is unidirectional, which means that the inner side of fabrics will still be wetted and attach to the body during the sweat transport process. To address these limitations, fruitful efforts have been made to improve the sweat transport through fabric, which drive water transport in a single direction by hierarchical structure design or surface chemical modification.(Su et al. 2016; Zhao et al. 2017) Many novel structures have been produced based on bionics, such as dragonfly wings,(Jones et al. 2020) spider silk,(Zheng et al. 2010) and cactus,(Ju et al. 2014) which exhibit directional water transport. In general, tree structures are composed of a proportional gradient in a particular direction. According to this, a biomimetic fabric with three layers of tree-shaped structure was reported, showing great moisture management performance.(Miao et al. 2018) In addition to traditional fabrics, many nanofibrous membranes (Fu et al. 2020)have been applied in directional water transport, (Zhang et al. 2020a)in which water can be transported from inner side to outer side. Another commonly used method is surface chemical modification, which forms energy gradient on the fabric surface to realize directional water transport. One useful strategy is Janus wettability,(Yang et al. 2016; Li et al. 2020; Mohammadi Ghaleni et al. 2020) which means that both side of fabric or membrane have completely different wettability. Janus wettability can be reached by surface modification technology, (Wang et al. 2018b)such as UV irradiation,(Zhu et al. 2020) 
plasma treatment,(Chen et al. 2018) and electrospray.(Ji et al. 2020; Liang et al. 2020; Zhang et al. 2020b) Consequently, hierarchical structure design and surface chemical modification consistently cooperate together to improve the differential capillary effect.

Owing to the promising application in moisture management textiles, directional water transport textiles have received increasing attention. Considerable achievements have been received. For example, Wang et al. (Wang et al. 2018c)proposed smart moisture wicking fabric with antigravity directional water transport and quick-dry performance based on biomimetic Murray membranes. Cai et al. (Fan et al. 2020)prepared a 3D transport fabric with advanced water transport property by coating discontinuous hydrophobic pattern on inner side and discontinuous superhydrophilic pattern on outer side. Qin et al.(Mao et al. 2019)fabricated one-dimensional (1D) fiber with controllable water transport performance by combining tree-like structure design and material optimization together. Unfortunately, few researches on synergistic effect of heat dissipation and water evaporation have been reported. Conventional fabrics such as cotton typically have low thermal conductivity.(Längauer et al. 2021) More importantly, the thermal conductivity of such fabrics can be regulated on a small scale by changing the fibrous density or structure. We adopt nano zinc oxide to increase the thermal conductivity of fabric without noticeably changes of fabric moisture and air permeability. High thermal conductivity not only improve heat transfer, but also accelerate sweat evaporation.

Herein, we fabricate a hydrophobic/hydrophilic Janus cotton fabric with asymmetric wettability for directional water transport by nano-ZnO treatment and single-side discontinuous hydrophobic screen-printing method. Significantly, the synergistic effect of the thermal conductivity performance and the differential capillary effects resulted in the outstanding directional water transport property of the obtained fabric. More vividly, the design of hydrophobic pattern which resembling river branch water transfer channel endows the cotton fabric with greatly improved water transport and diffusion capacity. As a result, the obtained cotton fabric was able to spontaneously pull the body-generated sweat away from the skin, thus providing an extremely dry and cool microclimate to the wearer, indicating an attractive prospect for the smart moisture wicking textiles.

\section{Experimental Section}

\section{Materials}

Nano Zinc oxide (nano-ZnO) and Cetyltrimethoxysilane were available from the Shanghai Macklin Biochemical Technology Co., Ltd. Triton was obtained from Shanghai Taitan Technology Co., Ltd. Titanate coupling agent was purchased from the Nanjing Chuangshi Reagent Co., Ltd. Thickener and adhesive were supplied by the Wuxi Feinuo Co., Ltd. Cotton fabric $\left(250 \mathrm{~g} \cdot \mathrm{m}^{-2}\right)$ was purchased from a local factory, the densities of cotton fabric is $280 \times 270$ (ends/10 cm).

\section{Preparation of Nano-Zno Dispersion}

$1.5 \%$ titanate coupling agent was dissolved in deionized water, and then $1.5 \%$ nano-ZnO particles and $1.5 \%$ dispersant Triton were added, and stirred it magnetically for 15 minutes. Finally, ultrasonic dispersion was performed for $1 \mathrm{~h}$ to obtain a dispersion solution of nano-ZnO, and then tested for particle size.

\section{Preparation of Nano-ZnO Cotton Fabric}

The cotton fabric was dipped into the nano-ZnO finishing solution, which was prepared by using titanate coupling agent and dispersant to disperse nano-ZnO solution under ultrasonication. The prepared fabric was immersed in 
the nano-ZnO finishing solution with a mass concentration of $20 \mathrm{~g} / \mathrm{L}$. Treat it in a constant temperature shaker at $30^{\circ} \mathrm{C}$ for $25 \mathrm{~min}$, and then proceed to the finishing process of drying $\left(80^{\circ} \mathrm{C}, 3 \mathrm{~min}\right)$ and baking $\left(150^{\circ} \mathrm{C}, 130 \mathrm{~s}\right)$. The dried fabric was used for UV resistance test.

\section{Preparation of Printed Cotton Fabric}

Cetyltrimethoxysilane (HDTMS) (2 wt\%), thickener (4 wt\%), pigment (1 wt\%) and deionized water (93 wt \%) were mixed under mechanical stirring (2000 rpm). Screen-printing technology was used to construct a discontinuous hydrophobic hexagon pattern with $5 \mathrm{~mm}$ side length on inner side of the cotton fabric. After drying $\left(80^{\circ} \mathrm{C}, 10 \mathrm{~min}\right)$ and baking $\left(150^{\circ} \mathrm{C}, 5 \mathrm{~min}\right)$, the obtained fabrics were washed thoroughly to remove the thickener, and then dried the sample at $80^{\circ} \mathrm{C}$. The printed cotton fabric is called discontinuous hydrophobic/hydrophilic Janus fabric.

\section{Characterization and Measurements}

\section{Fourier transform infrared (FT-IR) spectra}

The Fourier transform infrared (FT-IR) spectra of the samples were monitored with a NICOLET is 10 transform

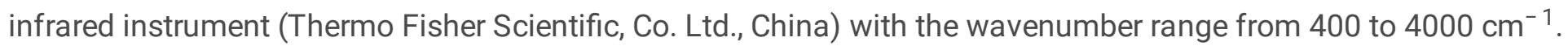

\section{XRD spectra}

The structures of the cotton fabric and nano-ZnO fabric were characterized by X-ray diffractometer from Japan over the $2 \theta$ range of $5-60^{\circ}$.

\section{Scanning electron microscopy (SEM)}

Scanning electron microscopy (SEM, SU1510, Hitachi) was used to observe the surface topography of cotton fabric, nano-ZnO fabric and discontinuous hydrophobic/hydrophilic Janus fabric.

\section{Energy dispersive spectroscopy (EDS)}

The elemental distribution of the nano-ZnO fabric was characterized by energy dispersive spectroscopy (EDS) on an EDAX-DX-4 energy dispersive analyzer.

\section{Contact angle (CA)}

The contact angle (CA) of the cotton fabric and nano-ZnO fabric measurements were carried out by a Drop Shape Analyzer 100 (Krüss, German) using liquid droplets of $5 \mu \mathrm{L}$ in volume. The size of nano-ZnO dispersion was tested with a nano particle size analyzer, and each sample was tested 3 times, and the test results were averaged.

\section{UV protection factor test}

The transmittance and UV protection factor (UPF) of nano-ZnO fabric was determined with a UV protection tester (Darong, YGB 912E, China).

\section{Temperature change test}

The temperature change of nano-ZnO fabric was recorded using a thermographic system (varioCAM hr head680, Infratec ESM GmbH). The photographs and videos were taken by a digital camera.

\section{Moisture management test}


The water transport property of discontinuous hydrophobic/hydrophilic Janus fabric was measured with a model M290 liquid moisture management tester from Hong Kong according to the GB/T 21655.2-2009 standard.

\section{Moisture permeability test}

According to GB/T 12704.1-2009 Determination of Fabric Moisture Permeability, moisture permeability of samples was measured to obtain the mass of water vapor passing vertically through a unit area sample within a specified time under a unit water vapor pressure difference.

\section{Air breathability test}

The air breathability of fabrics was tested before and after single-sided discontinuous hydrophobic screen-printing. Each piece of fabric was measured 10 times on different positions, the average value was recorded.

\section{Results And Discussion Schematic diagram}

In order to prepare uniform and stable nano-ZnO dispersion, the dispersant triton and titanate coupling agent were mixed with the nano-ZnO solution, and then the mixture was ultrasonically emulsified for $1 \mathrm{~h}$. Cotton fabric was finished with nano-ZnO dispersion by impregnating to improve the thermal conductivity of fabric. As revealed by particle size analysis, the size of nano-ZnO dispersion varied from 300 to $800 \mathrm{~nm}$, and the average nano particle size was $458 \mathrm{~nm}$ (Fig. 1a). The PDI (dispersion index) was a uniform and stable dispersion of 0.159. As shown in Fig. 1b, SEM images of nano-ZnO, which shows the uniformity of the nano-ZnO particles.

Then, single-sided discontinuous hydrophobic finishing was performed on the cotton fabric by screen printing. The resulted fabrics with a resembling river branches hexagon water-transfer-channel which greatly improved the water transport performance of the fabric (Fig. 1d). Figure 1c illustrates the preparation process of discontinuous hydrophobic/hydrophilic Janus fabric. The improvement of the thermal conductivity of cotton fabric will accelerate the evaporation of sweat on the surface of cotton fabric, which is beneficial to the improvement of the thermal and wet comfort of the fabric. As the preparing method is easy to practice, it contributes a novel approach to the fabrication of directional water transport textiles.

\section{Characterization of Nano-Zno Cotton Fabric}

A commercial cotton fabric was used as the substrate due to its suitable thickness and denseness. According to the SEM image (Fig. 2a, b), the surface of cotton fibric is smooth (Fig. 2a). As shown in Fig. 2b, the cotton fiber is covered by a layer of nano-ZnO particles, which increases the roughness of the cotton fiber, indicating the success in loading the nano-ZnO particles onto the cotton fabric. The chemical compositions of the nano-ZnO coated cotton fabric surface were examined through the EDS analysis. As shown in Fig. 2(c-e), elemental mappings of the nano$\mathrm{ZnO}$ cotton fabric show that the distribution of $\mathrm{C}$ and $\mathrm{Zn}$ is uniform across the whole nano-ZnO cotton fabric surface, demonstrating that nano-ZnO are well dispersed on the cotton fabric.

As displayed in Fig. 2f, FT-IR spectrogram of cotton fabric and nano-ZnO cotton fabric. The peak at $3337 \mathrm{~cm}^{-1}$ was derived from $-\mathrm{OH}$ stretching vibration, and $1168 \mathrm{~cm}^{-1}, 1104 \mathrm{~cm}^{-1}, 1058 \mathrm{~cm}^{-1}$, and $1023 \mathrm{~cm}^{-1}$ appeared in the spectrum of cotton fabric were derived from -C-O-C- stretching vibration, which constitute the characteristic absorption peaks of cotton fabric. After using titanate coupling agent to finish cotton fabric with nano-ZnO, two new characteristic peaks appeared.(Liu et al. 2012) The bands at $1718 \mathrm{~cm}^{-1}$ were associated with stretching 
vibration of phospholipid bond formed by the reaction of the coupling agent and the -OH on the cotton fabric,(Salla et al. 2012) respectively. In addition, the absorption peak around $816 \mathrm{~cm}^{-1}$ is corresponding to the bending vibration of the $\mathrm{C}-\mathrm{H}$ in the para-substituted benzene ring of the coupling agent, indicating that nano-ZnO was loaded in the cotton fabric. The crystalline structures of nano-ZnO fabric were identified by X-ray diffraction (XRD). The XRD patterns of nano-ZnO fabric are described in Fig. $2 \mathrm{~g}$. Cotton fabric has corresponding cellulose I structure at $2 \theta=14.89^{\circ}, 16.39^{\circ}$, and $22.62^{\circ}$ characteristic diffraction peaks. After finishing with nano-Zno, not only the characteristic diffraction peaks of cellulose I-type structure appeared on the cotton fabric at $2 \theta=14.89^{\circ}, 16.39^{\circ}$, $22.62^{\circ}$, but also nano-ZnO (100), (101), (110) crystal planes appeared at $2 \theta=31.79^{\circ}, 36.37^{\circ}, 56.67^{\circ}$, (Wang et al. 2017, 2018a) which proved that nano-ZnO was finished on the cotton fabric.

\section{Wettability of Nano-ZnO Cotton Fabric and Printed Cotton Fabric}

The moisture wicking performance of the fabrics were enhanced by adjusting the surface wettability. Through the video obtained from the experiment, we observed and compared the water droplet wetting of cotton fabric and nano-ZnO cotton fabric fromo s to $0.15 \mathrm{~s}$. The water contact angle of cotton fabric and nano-ZnO cotton fabric are measured, and the results are shown in Fig. 3(a-c). It is shown that cotton fabric has strong hydrophilicity. In Fig. 3c, it can be seen that the cotton fabric after nano-ZnO pretreatment also has strong hydrophilicity, indicating that nano-ZnO pretreatment will not affect its hydrophilicity, and will not affect the next step of discontinuous hydrophobic printing.

Through single-sided discontinuous hydrophobic screen-printing method, the inner side (the side contacting the skin) is partially hydrophobic and the outer side (the side that does not touch the skin) is hydrophilic. In the same way, the wettability of printed cotton fabric was observed and compared with the video obtained from the experiment. Water contact angle of discontinuous hydrophobic area and discontinuous hydrophilic area are measured, and the results are shown in Fig. 3(d-f). Cotton fabric after hydrophobic treatment possess extremely strong hydrophobicity while other parts without hydrophobically finishing have strong hydrophilicity. As shown in Fig. $3 e$, water contact angle of those hydrophilic parts decreases dramatically to $0^{\circ}$ in the first $0.2 \mathrm{~s}$. On the contrary, cotton fabric after hydrophobic treatment remains $130^{\circ}$ in the first $0.2 \mathrm{~s}$ and decrease slightly in 1 minute or even longer. Single-sided discontinuous hydrophobic screen-printing makes the inner and outer layers of the cotton fabric own different moisture absorption, which provide a differential capillary effect and realize the unidirectional moisture transport function of the cotton fabric. The sweat produced by the pores of the human body transfers from the inner surface of the cotton fabric to the outer surface and evaporates rapidly. Thus, discontinuous hydrophobic screen-printing fabric possess the ability of one-way water transport.

\section{Moisture Wicking Property of Printed Cotton Fabric}

Regarding the fact that different hydrophilic-hydrophobic proportion of the cotton fabric surface would affect the transfer speed of sweat and the one-way water transport ability, the screen-printing patterns with different hydrophilic and hydrophobic ratios are designed. More vividly, once the sweat excreted from human skin touches the inner side, it transfers along the hydrophilic area preferentially which represents the gap width of hexagon water-transfer-channel, just like a river branch. In the discussion of hydrophobic screen-printing section, $\mathrm{C}$ stands for cotton fabric, C-Z stands for cotton fabric finished only with nano-ZnO, and C-X\% stands for cotton fabrics with single-sided discontinuous hydrophobic treatment, C-Z-25\%, C-Z-50\%, and C-Z-75\% respectively represent the cotton fabric treated with single-sided discontinuous hydrophobic treatment after nano-ZnO finishing ( $\mathrm{X} \%$ is the percentage of hydrophilic area to hydrophobic area). C-Z-H represents the cotton fabric treated with single-sided continuous hydrophobic treatment after nano-ZnO finishing. 
Moisture wicking functionality of discontinuous hydrophobic/hydrophilic Janus fabric was tested by M290 liquid moisture management tester, and the results are shown in Table 1. Wetting time is the time when the fabric starts to be wetted. The higher the hydrophilicity of the cotton fabric, the faster it will be wetted. Since cotton fabrics inherently have good hydrophilicity, the wetting time of the fabrics before and after hydrophobic finishing is relatively fast. The wetting time of C-Z, C-Z-75\%, C-Z-50\%, C-Z-25\% and C-Z-H, indicates that the fabric has the ability to quickly absorb moisture. The water absorption rate also reflects the ability of the cotton fabric to absorb water. For C-Z-75\%, C-Z-50\%, C-Z-25\% and C-Z-H, the top surface shows lower water absorption rate than the bottom surface, this is because C-Z-75\%, C-Z-50\%, C-Z-25\%, C-Z-H have undergone hydrophobic treatment, and the treated hydrophobic area make the water absorption rate of the fabric decline. According to the water absorption rate, single-sided discontinuous hydrophobic screen-printing fabric has a better ability to absorb moisture. The max wetted radius reflects the diffusion capacity of moisture in the cotton fabric. In comparison with cotton fabric, the max wetted radius of C-Z-75\%, C-Z-50\%, C-Z-25\%, C-Z-H have been improved. The spreading speed of liquid water is the diffusion rate of fabric when wetting to the maximum wetted radius, which reflects the rapid drying ability of the cotton fabric. The different spreading speed of top surface and bottom surface indicate that the cotton fabric can quickly evaporate water, which is beneficial to moisture transfer and evaporation in the air. Comparing results of CZ-75\%, C-Z-50\%, C-Z-25\%, all the samples have the directional water transport property. Moreover, it was worth mentioning that C-Z-50\% has the largest difference in spreading speed between the top surface and bottom surface which implying that sample C-Z-50\% has a preferable directional water transport property. The one-way transport capability is the ratio of the water content difference between the top surface and bottom surface to the total test time, which reflects the directional water transport property directly. Cotton fabric does not possess a directional water transport property with the one-way transmission index of 19.58. After single-side continuous hydrophobic finishing, the one-way transmission index increases to 45.97. However, C-Z-75\%, C-Z-50\%, C-Z-25\% reach 94.35 , $147.26,63.57$ respectively. As for C-Z-50\%, the one-way transmission index was highest impling that C-Z-50\% possess an enhanced directional water transport property.

Table 1

Moisture management test of cotton fabric with different hydrophilic and hydrophobic ratios

\begin{tabular}{|c|c|c|c|c|c|c|c|c|c|}
\hline \multirow[t]{2}{*}{ Samples } & \multicolumn{2}{|c|}{ Wetting times(s) } & \multicolumn{2}{|c|}{$\begin{array}{l}\text { Absorption } \\
\text { rate(\%/s) }\end{array}$} & \multicolumn{2}{|c|}{$\begin{array}{l}\text { Max wetted } \\
\text { radius(mm) }\end{array}$} & \multicolumn{2}{|c|}{$\begin{array}{l}\text { Spreading } \\
\text { Speed(mm/s) }\end{array}$} & \multirow{2}{*}{$\begin{array}{l}\text { One way } \\
\text { transport } \\
\text { capability }\end{array}$} \\
\hline & $\begin{array}{l}\text { Top } \\
\text { Surface }\end{array}$ & $\begin{array}{l}\text { Bottom } \\
\text { Surface }\end{array}$ & $\begin{array}{l}\text { Top } \\
\text { Surface }\end{array}$ & $\begin{array}{l}\text { Bottom } \\
\text { Surface }\end{array}$ & $\begin{array}{l}\text { Top } \\
\text { Surface }\end{array}$ & $\begin{array}{l}\text { Bottom } \\
\text { Surface }\end{array}$ & $\begin{array}{l}\text { Top } \\
\text { Surface }\end{array}$ & $\begin{array}{l}\text { Bottom } \\
\text { Surface }\end{array}$ & \\
\hline C-Z & 2.044 & 0.403 & 27.22 & 22.79 & 15 & 15 & 3.48 & 11.62 & 19.58 \\
\hline C-Z-75\% & 0.325 & 0.325 & 23.98 & 25.34 & 10 & 10 & 2.01 & 12.93 & 94.35 \\
\hline C-Z-50\% & 6.575 & 0.325 & 16.41 & 22.19 & 10 & 10 & 1.31 & 12.78 & 147.26 \\
\hline C-Z-25\% & 3.528 & 0.325 & 23.85 & 26.54 & 10 & 15 & 1.96 & 13.26 & 63.57 \\
\hline $\mathrm{C}-\mathrm{Z}-\mathrm{H}$ & 2.747 & 0.325 & 22.72 & 25.46 & 10 & 10 & 2.26 & 12.87 & 45.97 \\
\hline
\end{tabular}

Suitable screen-printing patterns make the fabric's directional water transport property better. When the hydrophobic area is less occupied, the cotton fabric does not have the directional water transport property. While the hydrophobic area is too large, its directional water transport property will be affected. As a result, it cannot transport the moisture from the inner surface of the cotton fabric to the outer surface quickly. In conclusion, it was obvious that hydrophobic pattern played strong part in improving the performance of directional water transport. 
The curves in Fig. 4 were the moisture content curves of the upper (inner) surface and the lower (outer) surface of different samples. The blue curve represents bottom (Outer side) surface while the green curve refers to the top (Inner side) surface. For the raw cotton fabric, the water content curves on both sides almost overlap, which means that the blank cotton fabric does not possess the performance of directional water transmission. It can be seen from Fig. 4(b-e) that the water content of the outer side has always been much higher than that of the inner side. It is supposed that water can transport more quickly from hydrophobic side to hydrophilic side. As a result, water will spread in multiple directions to increase the diffusion area. The upper surface moisture content of C-Z-50\% increased remarkably to $517 \%$ in the first 20 s, and then gradually decreased to $450 \%$. But the maximum moisture content of the bottom surface was $330 \%$, which was lower than that of the upper surface. The great difference of moisture content between upper surface and bottom surface implied that C-Z-50\% possessed an improved directional water transport property, which is consistent with the data in the Table 1.

The moisture permeability results of cotton fabrics with different hydrophilic/hydrophobic ratios are shown in Table 2. The result of cotton fabric is $326.61 \mathrm{~g} /\left(\mathrm{m}^{2} \square \mathrm{h}\right)$. With the hydrophobic screen-printing area increases, the moisture permeability of the printed cotton fabric gradually decreases. Due to the large number of hydroxyl groups on the cellulose molecule, the cotton fabric has a higher moisture regain rate and good moisture absorption performance. After finishing with hydrophobic agent, some of the hydroxyl groups on the cellulose molecules are blocked, resulting in a drop of moisture regain. Compared to the best directional water transport property of C-Z$50 \%$, the slight change in moisture permeability can be ignored.

Table 2

Moisture permeability test of cotton fabric with different hydrophilic and hydrophobic ratios

\begin{tabular}{|lllll|}
\hline Samples & $\begin{array}{l}\text { Weight before moisture } \\
\text { absorption } \mathrm{M}_{\mathbf{1}} / \mathbf{g}\end{array}$ & $\begin{array}{l}\text { Weight after moisture } \\
\text { absorption } \mathrm{M}_{\mathbf{2}} / \mathbf{g}\end{array}$ & $\begin{array}{l}\text { Weight } \\
\text { difference }(\mathrm{g})\end{array}$ & $\begin{array}{l}\text { Moisture } \\
\text { permeability } \\
\mathbf{g} /\left(\mathbf{m}^{2} \cdot \mathbf{h}\right)\end{array}$ \\
\hline C-Z & 154.641 & 155.564 & 0.923 & 326.61 \\
\hline C-Z-75\% & 154.862 & 155.769 & 0.907 & 320.95 \\
\hline C-Z-50\% & 155.150 & 156.045 & 0.895 & 316.70 \\
\hline C-Z-25\% & 155.481 & 156.369 & 0.888 & 314.23 \\
\hline C-Z-H & 153.789 & 154.606 & 0.817 & 289.10 \\
\hline
\end{tabular}

With respect to the air permeability, cotton fabric reaches $206.70 \mathrm{~mm} / \mathrm{s}$. As the ratio of hydrophilic and hydrophobic areas decrease, the hydrophilic area decreases, and the air permeability of printed cotton fabric reduces correspondingly (Fig. 5b). This is due to the hydrophobic finishing of the cotton fabric affect its air permeability. Compared to the best directional water transport property of C-Z-50\%, the slight change in air permeability can be ignored.

\section{Thermal Property of Printed Cotton Fabric}

The thermal conductivity of printed cotton fabric was tested by heating pad and FLIR infrared camera. The heating pad was set at $39^{\circ} \mathrm{C}$ to ensure that the fabric on the heating pad was maintained at about $37^{\circ} \mathrm{C}$. The self-made microenvironment was used to simulate the microclimate of human skin. The temperature of cotton fabric and printed cotton fabric were measured with a FLIR infrared camera every $10 \mathrm{~s}$ until the temperature of the sample was maintained in a stable temperature range. 
As shown in Fig. 6(b, c), printed cotton fabric heats up faster than the cotton fabric because the nano-ZnO possess certain thermal conductivity. Simulating the human skin environment, printed cotton fabric owns a higher heat absorption rate. As shown in Fig. 6a, the temperature change of two curves after $40 \mathrm{~s}$ is not obvious, because the two fabric has reached a balance with the surrounding thermal environment. However, printed cotton fabric obtains a higher surface temperature after the balance, which also proves the improvement of the thermal conductivity of the cotton fabric after nano-ZnO finishing. On the other hand, it means that compared with the cotton fabric, printed cotton fabric can take away more heat from the skin through thermal conduction in the same time.

\section{UV Protective Property of Nano-ZnO Cotton Fabric}

Nano-ZnO has good ultraviolet resistance, thus nano-ZnO cotton fabric possess certain UV protective property. Through UV resistance test of nano-ZnO fabric, the UPF (ultraviolet protection factor value), UVA (sunlight ultraviolet radiation with wavelength of $315 \mathrm{~nm}-400 \mathrm{~nm}$ ) and UVB (sunlight ultraviolet radiation with wavelength of $280 \mathrm{~nm}-315 \mathrm{~nm}$ ) transmittance are analyzed (Fig. 7). The results show that the UV transmittance of cotton fabric is higher, which indicates that the cotton fabric has no UV protective property. As the content of nano-ZnO in dispersion increases gradually, the UV transmittance values of the nano-ZnO cotton fabric decreases correspondingly. When the content of nano-ZnO in dispersion reaches to $20 \mathrm{~g} / \mathrm{L}$, the UV protection factor (UPF) values of cotton fabric maintain a certain range of balance, which means the adsorption of cotton fabric and nano$\mathrm{ZnO}$ particles is saturated. In order to avoid unnecessary consumption, the optimal mass concentration of nano$\mathrm{ZnO}$ is $20 \mathrm{~g} / \mathrm{L}$. Nano-ZnO has the function of scattering and absorbing ultraviolet rays. When irradiated by ultraviolet rays in sunlight, electrons in the valence band of $\mathrm{ZnO}$ are excited, and hole-electron pairing effects will occur. Thus, it has the function of absorbing ultraviolet rays. Compared to the wavelength of ultraviolet light, the particle size of nano-ZnO is very small, so it can scatter ultraviolet light in all directions. This scattering law of ultraviolet light conforms to the Raylieigh light scattering law. Nano-ZnO not only has good thermal conductivity, but also has certain UV protective property, which makes nano-ZnO play an important role in the development of functional textiles.

\section{Conclusions}

In summary, discontinuous hydrophobic/hydrophilic Janus fabric with asymmetric wettability was prepared by nano-ZnO pretreatment and single-side discontinuous hydrophobic screen printing. The design of hydrophobic pattern resembling river branches hexagon water-transfer-channel which greatly improved the water transport and diffusion performance of the fabric. Under the combined action of the hydrophobic pattern and the hydrophilic region, the discontinuous hydrophobic/hydrophilic Janus fabric possesses a higher directional water transport property of $147.26 \%$, and an excellent UPF of 145 for UV protection. Moreover, it is worth mentioning that printed cotton fabric possesses a desired thermal conductivity, which is beneficial to the improvement of directional water transport property. This unique concept may have inspired the design of moisture-absorbing, breathable and sports textiles, making it promising for the application of smart textiles.

\section{Declarations}

Acknowledgement This study was financially supported by the National Natural Science Foundation of China (21975107), Natural Science Foundation of Jiangsu Province (SBK2019020945) and Fundamental Research Funds for the Central Universities (JUSRP51724B).

Conflict of interest The authors have no conflicts of interest to declare that are relevant to the content of this article. 
Ethical approval This article does not contain any studies with human participants or animals performed by any of the authors. In this experiment, we did not collect any samples of human and animals.

Informed consent Informed consent was obtained from all individual participants included in the study.

\section{References}

1. Bai H, Ju J, Zheng Y, Jiang L (2012) Functional fibers with unique wettability inspired by spider silks. Adv Mater 24:2786-2791. https://doi.org/10.1002/adma.201200289

2. Chen X, Porto C, Lo, Chen Z et al (2018) Single step synthesis of Janus nano-composite membranes by atmospheric aerosol plasma polymerization for solvents separation. Sci Total Environ 645:22-33. https://doi.org/10.1016/j.scitotenv.2018.06.343

3. Fan Z, Zhao H, Zhou M et al (2020) Three-dimensional transport fabrics with ultrafast water transporting and diffusion inspired by river-diversion. Mater Lett 262:127050. https://doi.org/10.1016/j.matlet.2019.127050

4. Fu C, Gu L, Zeng Z, Xue Q (2020) Simply Adjusting the Unidirectional Liquid Transport of Scalable Janus Membranes toward Moisture-Wicking Fabric, Rapid Demulsification, and Fast Oil/Water Separation. ACS Applied Materials Interfaces 12:51102-51113. https://doi.org/10.1021/acsami.0c15158

5. He X, Yang S, Pei Q et al (2020) Integrated Smart Janus Textile Bands for Self-Pumping Sweat Sampling and Analysis. ACS Sensors 5:1548-1554. https://doi.org/10.1021/acssensors.0c00563

6. Ji X, Li R, Jia W et al (2020) Co-Axial Fibers with Janus-Structured Sheaths by Electrospinning Release Corn Peptides for Wound Healing. ACS Applied Bio Materials 3:6430-6438.

https://doi.org/10.1021/acsabm.0c00860

7. Jones C, Wortmann FJ, Gleeson HF, Yeates SG (2020) Textile materials inspired by structural colour in nature. RSC Advances 10:24362-24367. https://doi.org/10.1039/d0ra01326a

8. Ju J, Zheng Y, Jiang L (2014) Bioinspired one-dimensional materials for directional liquid transport. Acc Chem Res 47:2342-2352. https://doi.org/10.1021/ar5000693

9. Kim HA (2020) Water/moisture vapor permeabilities and thermal wear comfort of the Coolmax®/bamboo/tencel included PET and PP composite yarns and their woven fabrics. J Text Inst 0:1-14. https://doi.org/10.1080/00405000.2020.1853409

10. Längauer M, Brunnthaller F, Zitzenbacher $G$ et al (2021) Modeling of the anisotropic thermal conductivity of fabrics embedded in a thermoplastic matrix system. Polym Compos 1-11. https://doi.org/10.1002/pc.25958

11. Li N, Yu C, Si Y et al (2018) Janus Gradient Meshes for Continuous Separation and Collection of Flowing Oils under Water. ACS Applied Materials Interfaces 10:7504-7511. https://doi.org/10.1021/acsami.8b00044

12. Li Z, Liang W, Li W et al (2020) Facile fabrication of a Janus mesh for water fluid unidirectional transportation. RSC Advances 11:1001-1011. https://doi.org/10.1039/d0ra08632k

13. Liang Y, Huang G, Zeng X et al (2020) Effects of hydrophilic layer on directional transport of water through robust tri-layered Janus fabrics prepared by electrospinning. Mater Lett 268:127583.

https://doi.org/10.1016/j.matlet.2020.127583

14. Liu HT, Zeng XF, Zhao H, Chen JF (2012) Highly transparent and multifunctional polymer nanohybrid film with superhigh ZnO content synthesized by a bulk polymerization method. Ind Eng Chem Res 51:6753-6759. https://doi.org/10.1021/ie300425v 
15. Mao N, Peng H, Quan Z et al (2019) Wettability Control in Tree Structure-Based 1D Fiber Assemblies for Moisture Wicking Functionality. ACS Applied Materials Interfaces 11:44682-44690. https://doi.org/10.1021/acsami.9b14370

16. Miao D, Huang Z, Wang X et al (2018) Continuous, Spontaneous, and Directional Water Transport in the Trilayered Fibrous Membranes for Functional Moisture Wicking Textiles. Small 14:1-10. https://doi.org/10.1002/smll.201801527

17. Mohammadi Ghaleni M, Tavakoli E, Bavarian M, Nejati S (2020) Fabricating Janus membranes via physicochemical selective chemical vapor deposition. AIChE J 66:1-6. https://doi.org/10.1002/aic.17019

18. Neves SF, Campos JBLM, Mayor TS (2017) Effects of clothing and fibres properties on the heat and mass transport, for different body heat/sweat releases. Appl Therm Eng 117:109-121. https://doi.org/10.1016/j.applthermaleng.2017.01.074

19. Parada M, Vontobel P, Rossi RM et al (2017) Dynamic Wicking Process in Textiles. Transp Porous Media 119:611-632. https://doi.org/10.1007/s11242-017-0901-5

20. Salla J, Pandey KK, Srinivas K (2012) Improvement of UV resistance of wood surfaces by using ZnO nanoparticles. Polym Degrad Stab 97:592-596. https://doi.org/10.1016/j.polymdegradstab.2012.01.013

21. Shou D, Fan J (2018) Design of Nanofibrous and Microfibrous Channels for Fast Capillary Flow. Langmuir 34:1235-1241. https://doi.org/10.1021/acs.langmuir.7b01797

22. Su B, Tian Y, Jiang L (2016) Bioinspired Interfaces with Superwettability: From Materials to Chemistry. J Am Chem Soc 138:1727-1748. https://doi.org/10.1021/jacs.5b12728

23. su Cl, Fang JX, Chen XH, wu WY (2007) Moisture Absorption and Release of Profiled Polyester and Cotton Composite Knitted Fabrics. Text Res J 77:764-769. https://doi.org/10.1177/0040517507080696

24. Wang H, Lin W, Qiu X et al (2018a) In Situ Synthesis of Flowerlike Lignin/ZnO Composite with Excellent UVAbsorption Properties and Its Application in Polyurethane. ACS Sustainable Chemistry Engineering 6:36963705. https://doi.org/10.1021/acssuschemeng.7b04038

25. Wang H, Niu H, Zhou H et al (2019) Multifunctional Directional Water Transport Fabrics with Moisture Sensing Capability. ACS Applied Materials Interfaces 11:22878-22884. https://doi.org/10.1021/acsami.9b06787

26. Wang H, Qiu X, Liu W et al (2017) A novel Lignin/ZnO hybrid nanocomposite with excellent UVAbsorption ability and its application in transparent polyurethane coating. Ind Eng Chem Res 56:11133-11141. https://doi.org/10.1021/acs.iecr.7b02425

27. Wang H, Wang W, Wang H et al (2018b) One-Way Water Transport Fabrics Based on Roughness Gradient Structure with No Low Surface Energy Substances. ACS Applied Materials Interfaces 10:32792-32800. https://doi.org/10.1021/acsami.8b08277

28. Wang X, Huang Z, Miao D et al (2018c) Biomimetic Fibrous Murray Membranes with Ultrafast Water Transport and Evaporation for Smart Moisture-Wicking Fabrics. ACS Nano. https://doi.org/10.1021/acsnano.8b08242

29. Wang Z, Wang Y, Liu G (2016a) Rapid and Efficient Separation of Oil from Oil-in-Water Emulsions Using a Janus Cotton Fabric. Angewandte Chemie - International Edition 55:1291-1294.

https://doi.org/10.1002/anie.201507451

30. Wang Z, Zhao J, Bagal A et al (2016b) Wicking Enhancement in Three-Dimensional Hierarchical Nanostructures. Langmuir 32:8029-8033. https://doi.org/10.1021/acs.langmuir.6b01864

31. Yang C, Han N, Han C et al (2019) Design of a Janus F-TiO2@PPS Porous Membrane with Asymmetric Wettability for Switchable Oil/Water Separation. ACS Applied Materials Interfaces 11:22408-22418.

Page $11 / 17$ 
https://doi.org/10.1021/acsami.9b05191

32. Yang HC, Hou J, Chen V, Xu ZK (2016) Janus Membranes: Exploring Duality for Advanced Separation. Angewandte Chemie - International Edition 55:13398-13407. https://doi.org/10.1002/anie.201601589

33. Zhang Q, Li Y, Yan Y et al (2020a) Highly Flexible Monolayered Porous Membrane with SuperhydrophilicityHydrophilicity for Unidirectional Liquid Penetration. ACS Nano 14:7287-7296. https://doi.org/10.1021/acsnano.0c02558

34. Zhang S, Huang J, Chen Z, Lai Y (2017) Bioinspired Special Wettability Surfaces: From Fundamental Research to Water Harvesting Applications. Small 13:1-28. https://doi.org/10.1002/smll.201602992

35. Zhang Y, Tian M, Wang L et al (2020b) Flexible Janus Textile-Based Electroosmotic Pump for Large-Area Unidirectional Positive Water Transport. Advanced Materials Interfaces 7:1-7. https://doi.org/10.1002/admi.201902133

36. Zhao Y, Wang H, Zhou H, Lin T (2017) Directional Fluid Transport in Thin Porous Materials and its Functional Applications. Small 13:1-22. https://doi.org/10.1002/smll.201601070

37. Zheng Y, Bai H, Huang Z et al (2010) Directional water collection on wetted spider silk. Nature 463:640-643. https://doi.org/10.1038/nature08729

38. Zhou H, Wang H, Niu H et al (2016) One-Way Water-Transport Cotton Fabrics with Enhanced Cooling Effect. Advanced Materials Interfaces 3:1-6. https://doi.org/10.1002/admi.201600283

39. Zhu R, Liu M, Hou Y et al (2020) Biomimetic Fabrication of Janus Fabric with Asymmetric Wettability for Water Purification and Hydrophobic/Hydrophilic Patterned Surfaces for Fog Harvesting. ACS Applied Materials Interfaces 12:50113-50125. https://doi.org/10.1021/acsami.0c12646

\section{Figures}


(a)

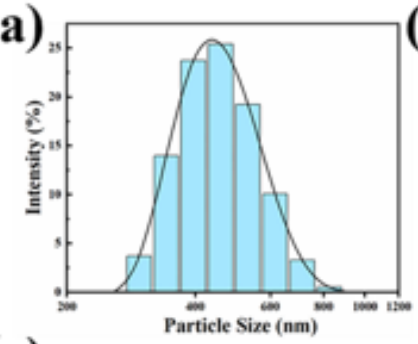

(c)
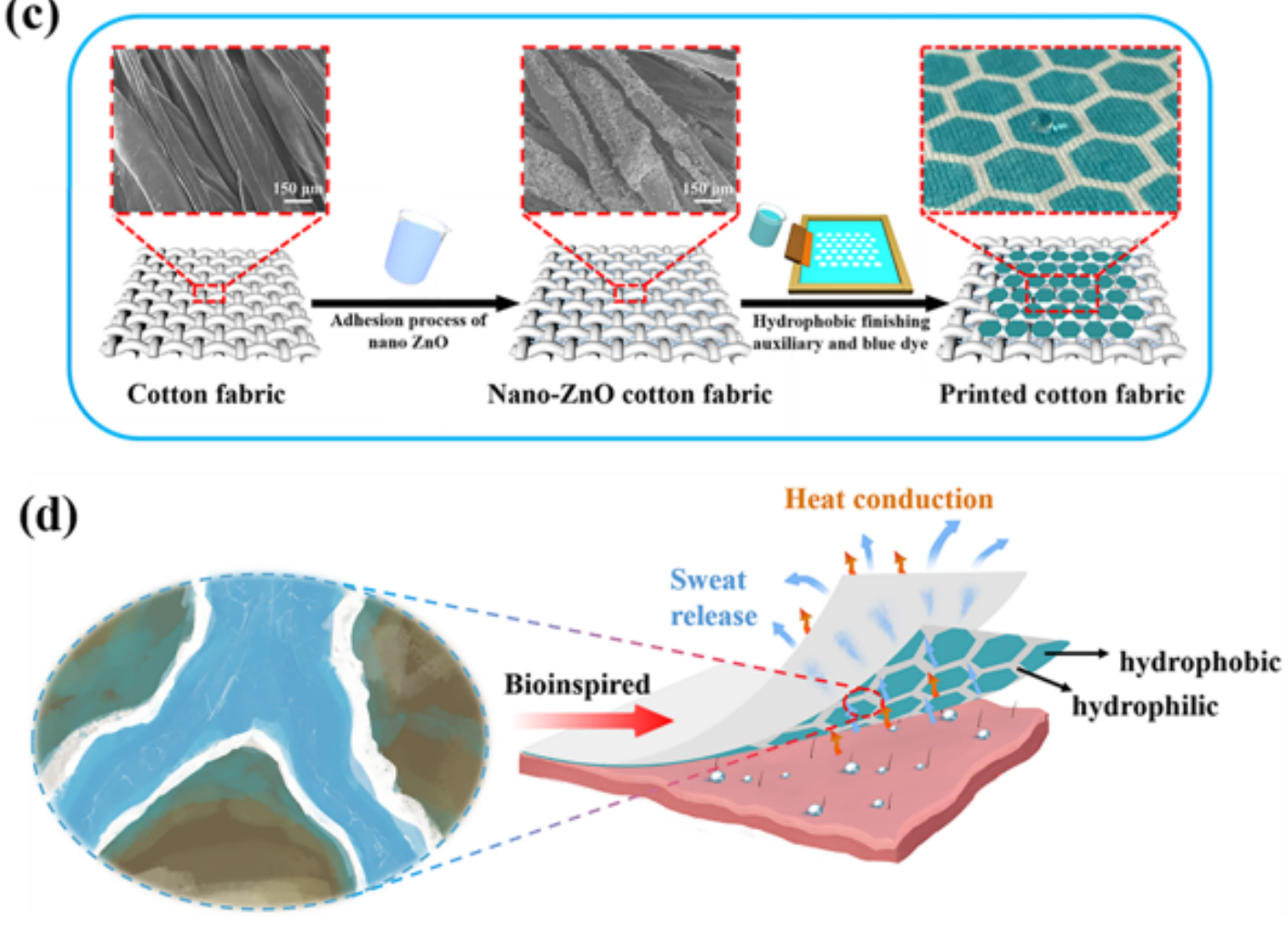

\section{Figure 1}

a Analysis of particle size of nano-ZnO dispersion. b SEM images of nano-ZnO. c Schematic diagram. $d$ The nature phenomenon of river branch (left) and schematic demonstration of the moisture wicking process (right).

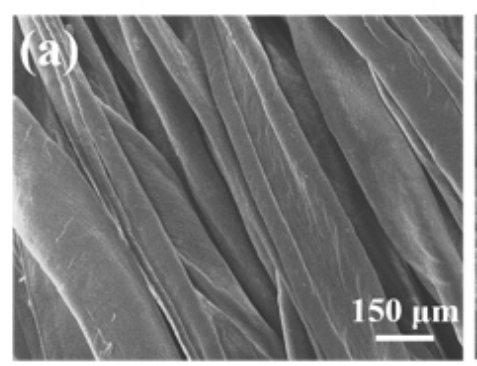

(e)

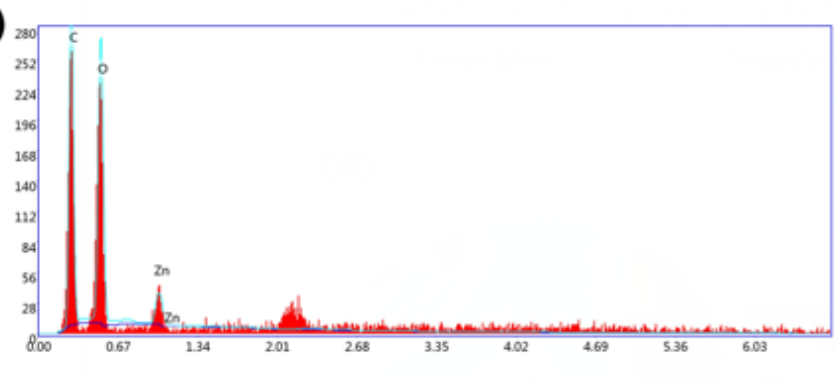

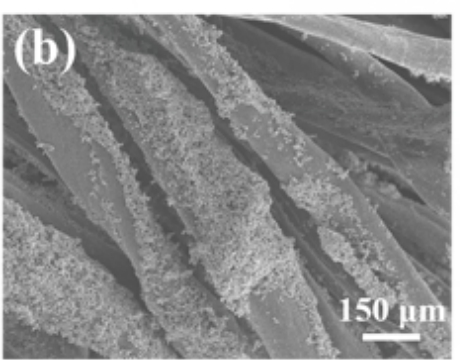

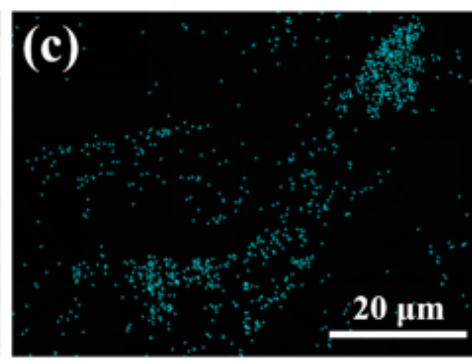

(f)

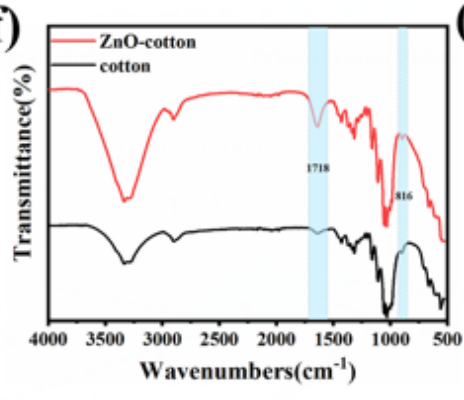

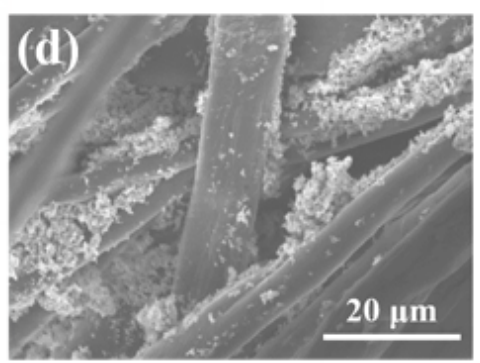

(g)

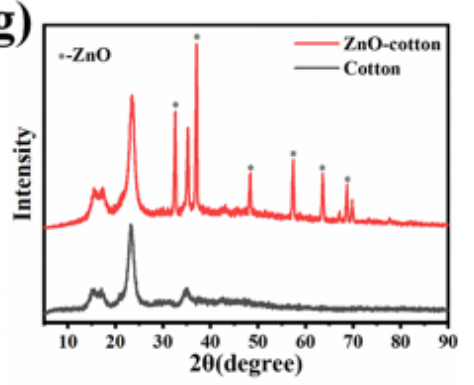


Figure 2

a SEM images of cotton fabric. b SEM images of nano-ZnO cotton fabric. c-e EDS of cotton fabric and nano-ZnO cotton fabric. f FTIR of cotton fabric and nano-ZnO cotton fabric. $\mathrm{g}$ XRD of cotton fabric and nano-ZnO cotton fabric.

(a)

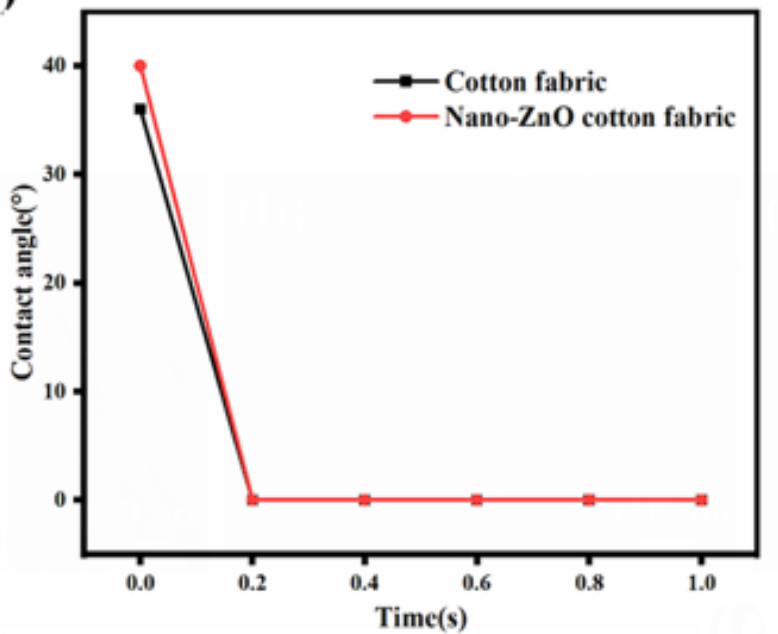

(b)

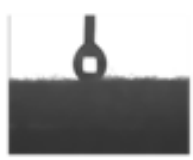

Os

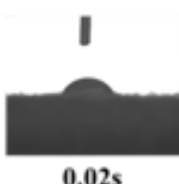

$0.02 \mathrm{~s}$

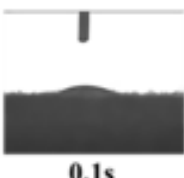

(c)

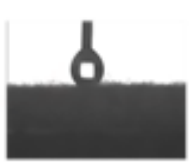

0s
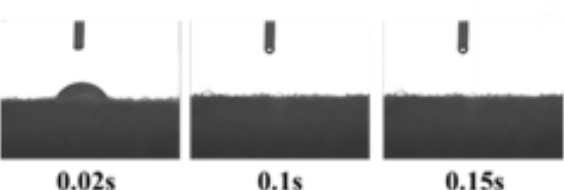

(d)

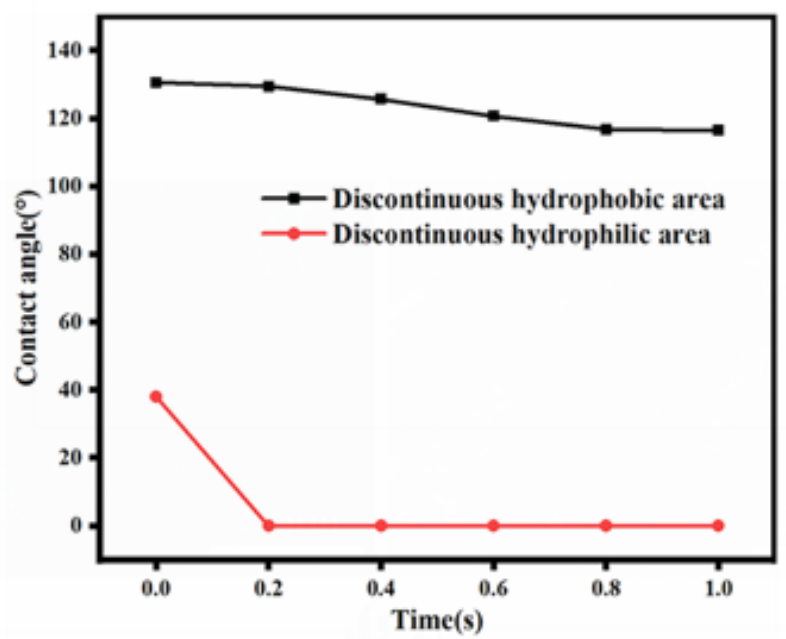

(e)
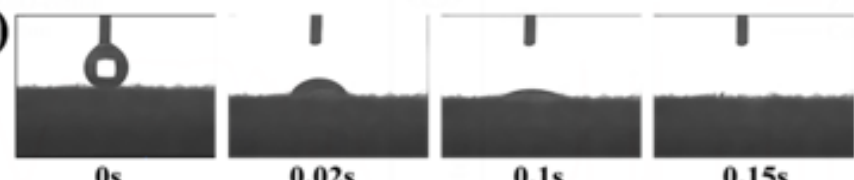

(f)

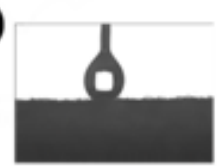

0s

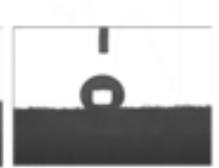

$0.3 \mathrm{~s}$

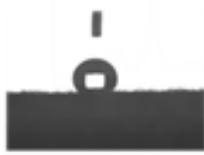

$0.6 \mathrm{~s}$

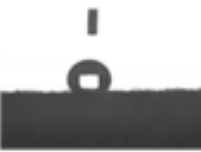

$1.0 \mathrm{~s}$

Figure 3

a Line chart of contact angle change. b Analysis of contact angle of cotton fabric. c Analysis of contact angle of nano-ZnO cotton fabric. $d$ Line chart of contact angle change. e Analysis of contact angle of discontinuous hydrophilic area. $f$ Analysis of contact angle of discontinuous hydrophobic area. 
(a)

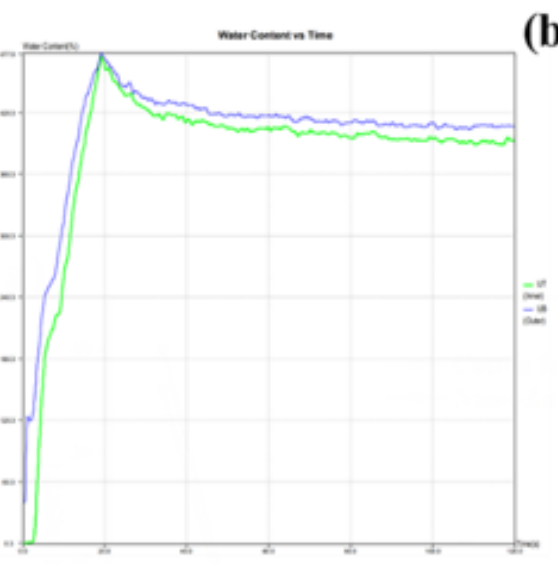

(d)

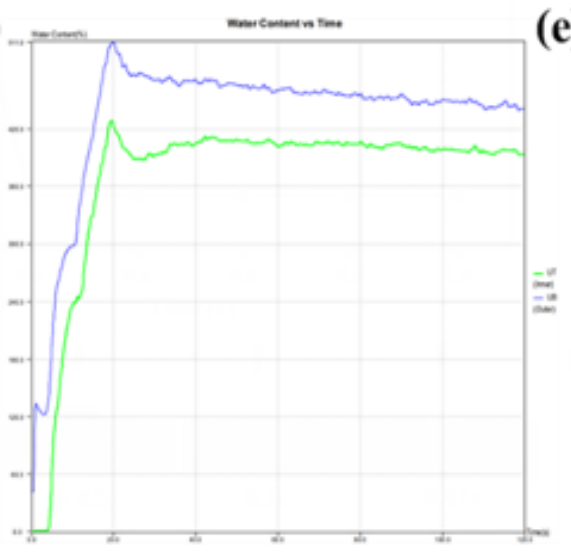

(b)

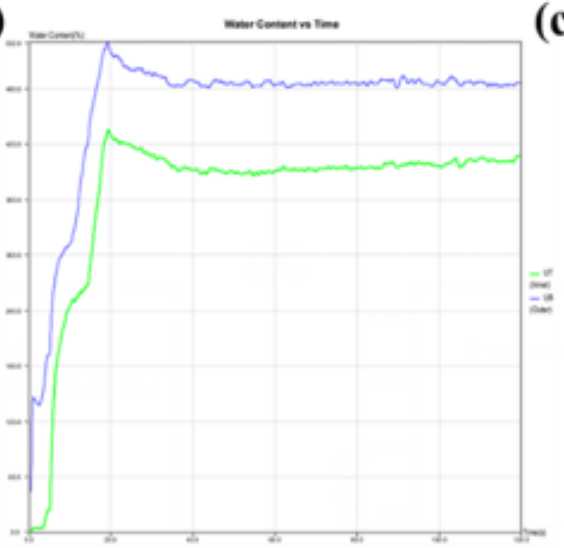

(e) (c)

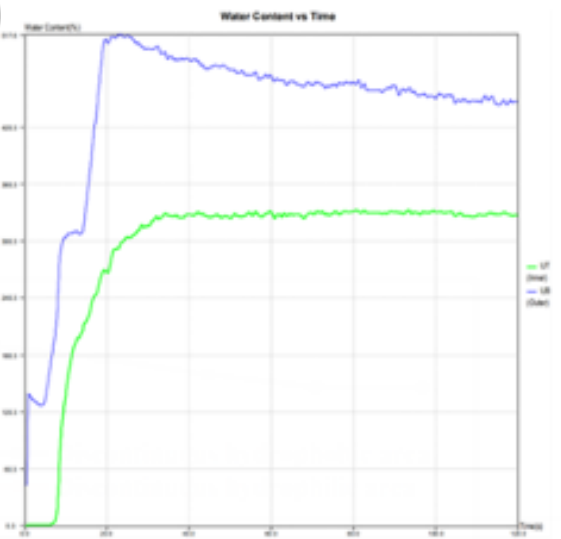

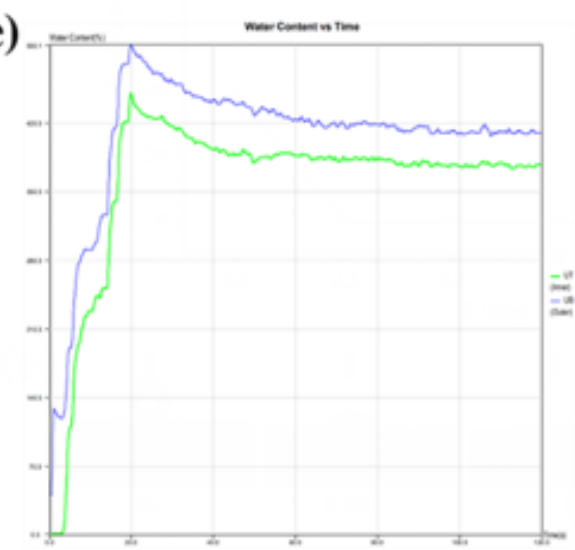

Figure 4

Moisture management test. a C-Z. b C-Z-75\%. c C-Z-50\%. d C-Z-25\%. e C-Z-H.

(a)

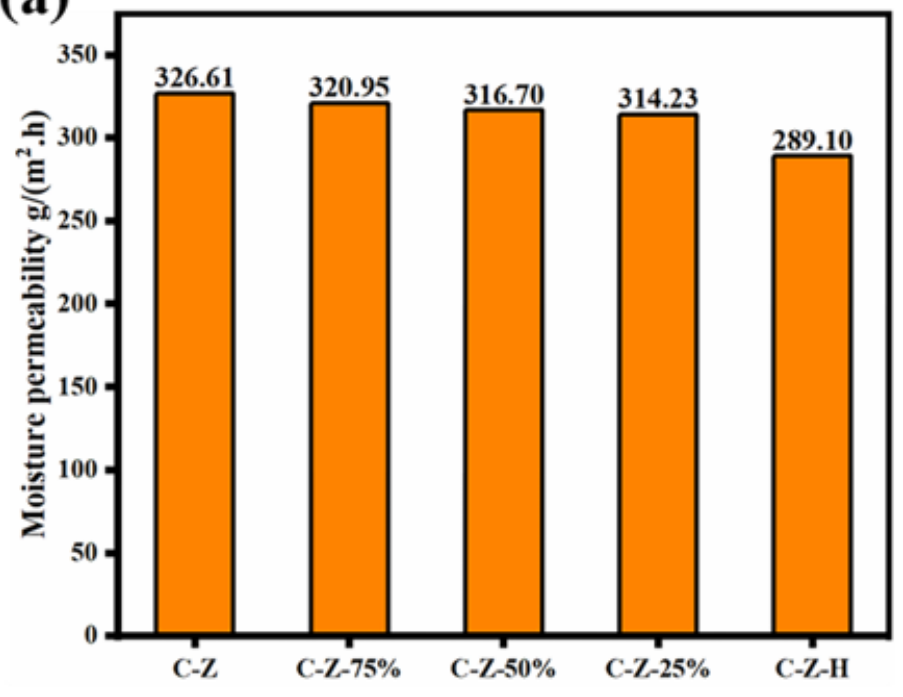

(b)

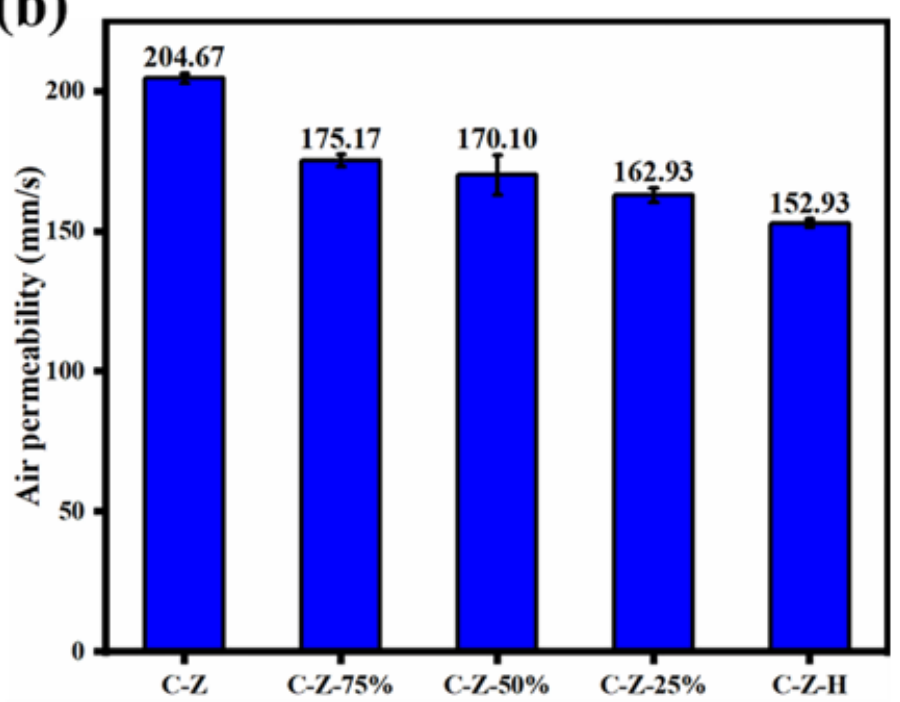

Figure 5

a Moisture permeability of printed cotton fabric. b Air permeability of printed cotton fabric. 

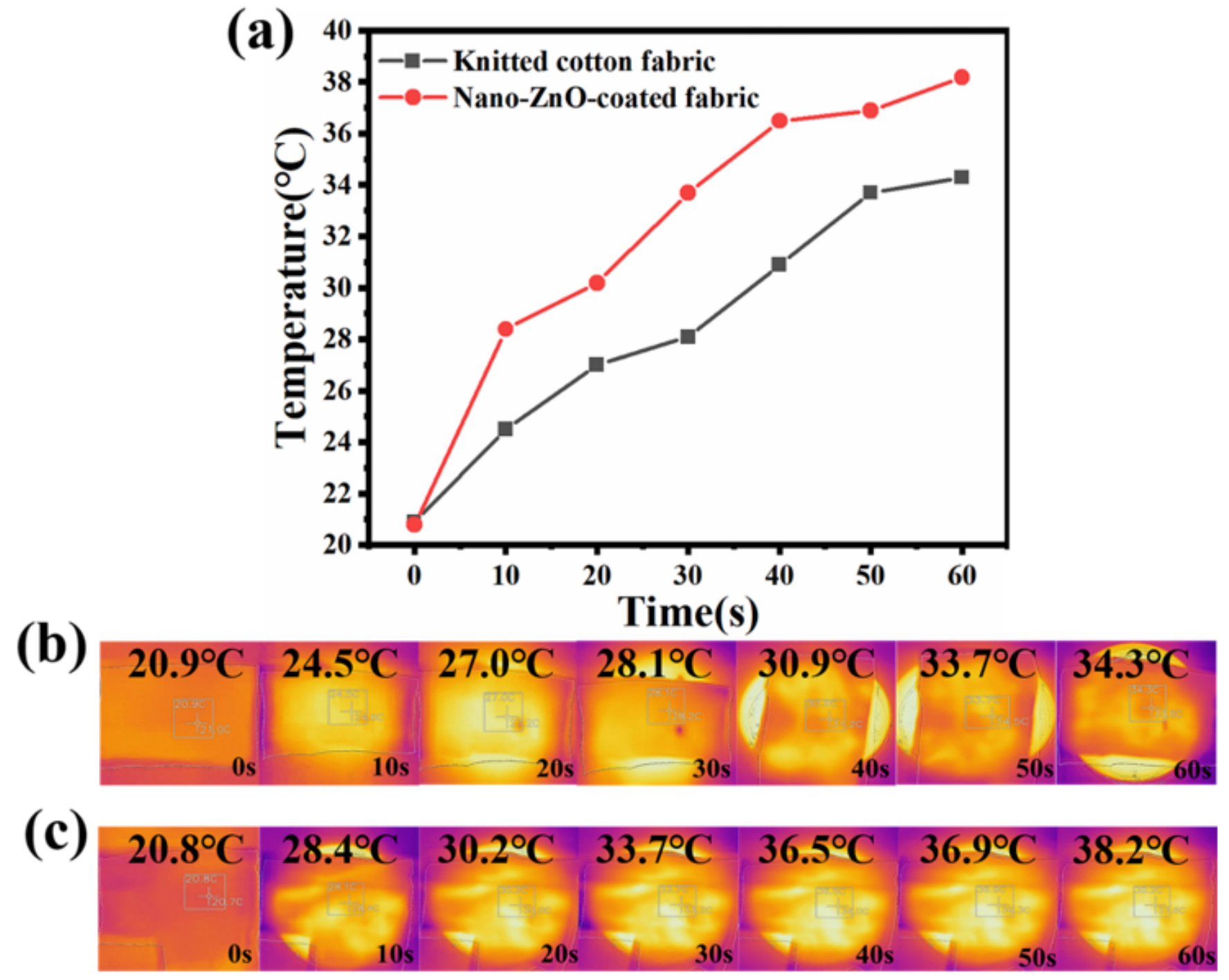

Figure 6

a Line chart of temperature change of cotton fabric and nano-ZnO cotton fabric in $60 \mathrm{~s}$. b IR schematic diagram of temperature change of cotton fabric. $\mathrm{c}$ IR schematic diagram of temperature change of nano-ZnO cotton fabric. 


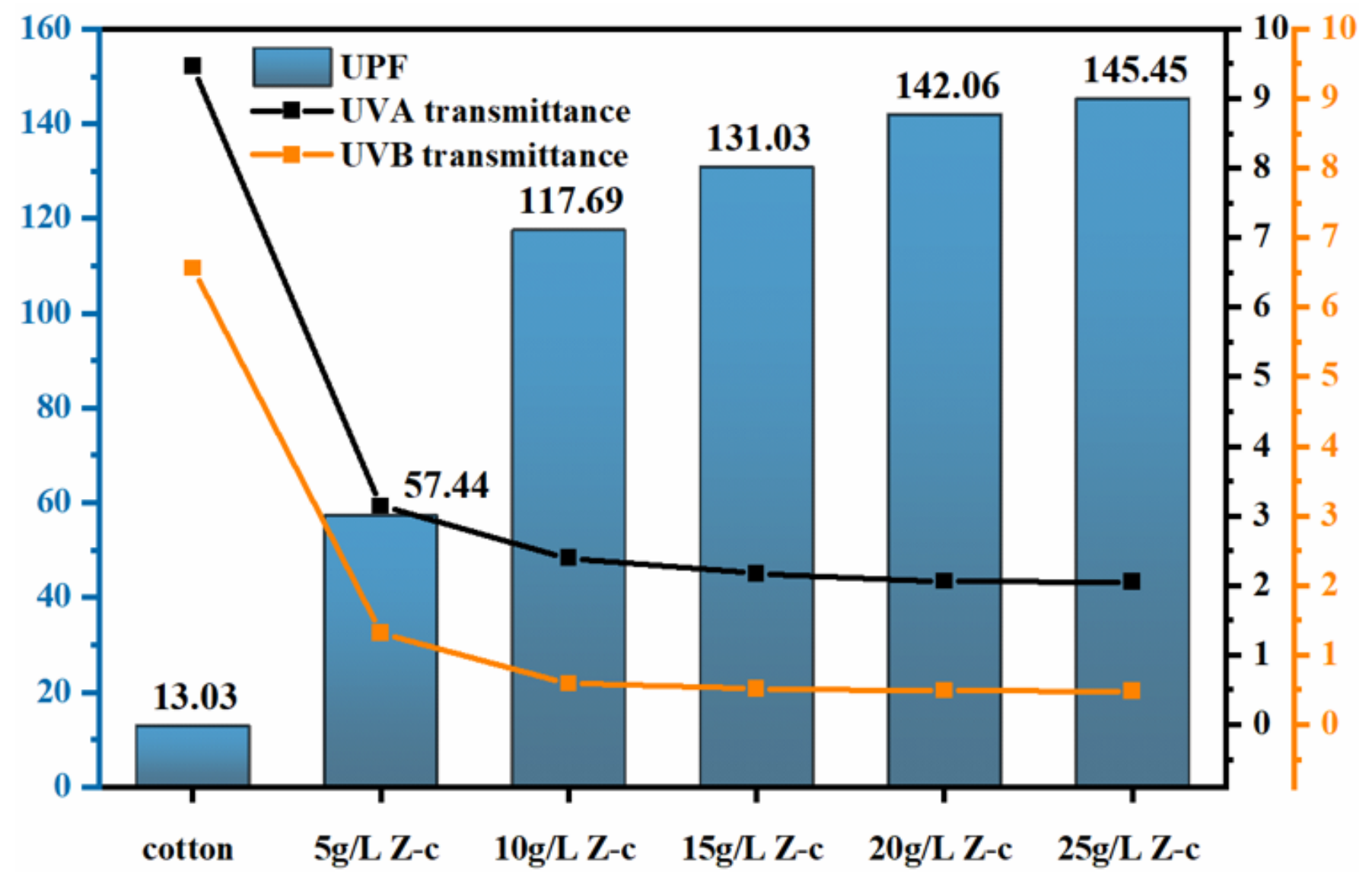

Figure 7

UV protective property of nano-ZnO cotton fabric with different nano-ZnO mass concentration.

\section{Supplementary Files}

This is a list of supplementary files associated with this preprint. Click to download.

- Graphicabstract.docx 